\title{
Vorwort zur 20. Auflage
}

Dieses Werk hat seinen Ursprung in den in der „Sammlung Göschen“ erschienenen Bänden Vermessungskunde I, II und III. Verfasser der 1. bis 9. Auflage des Bandes I, der 1. bis 7. Auflage des Bandes II und der 1. bis 6. Auflage des Bandes III (1910 - 1949) war Prof. Dr.-Ing. Paul Werkmeister. Von 1959 - 1982 setzte Prof. Dr.Ing. Walter Großmann die Neubearbeitung fort, für den Band I bis zur 15. Auflage, den Band II bis zur 12. Auflage und den Band III bis zur 11. Auflage. Seit 1983 übernahm Prof. Dr.-Ing. Heribert Kahmen die Weiterbearbeitung der Bände, die zu diesem Zeitpunkt schon eine Geschichte und Tradition von mehr als siebzig Jahren aufweisen konnten. Er bearbeitete den Band I bis zur 17., den Band II bis zur 14. und den Band III bis zur 12. Auflage.

Ab 1993 wurden die drei Bände zu einem gemeinsamen Werk zusammengefasst, das 1993 als 18. Auflage und 1997 als 19. Auflage erschienen ist. Die nun neu vorgelegte 20. Auflage umfasst 19 Kapitel. Weitgehende Neuorientierungen und Schwerpunktverschiebungen machten erneut eine Neugliederung des Stoffgebietes notwendig.

Seit der ersten Auflage befasst sich das Werk mit der Thematik, wie sich mit unterschiedlichsten Messsystemen, die sich in Satelliten, Flugzeugen, Schiffen oder auf der Erde befinden, Informationen über die Gestalt der Erdoberfläche sammeln lassen und wie diese in Referenzsystemen (Koordinatensystemen) dargestellt werden können, um sie einer Vielzahl interessierter Nutzer zur Verfügung zu stellen. Rasante Fortschritte in verschiedenen Bereichen wie Satelliten-, Laser-, Computertechnologien bedingen nicht nur eine ständige Erneuerung der Stoffgebiete, sondern auch interessante Ausweitungen der Anwendungen in anderen Ingenieur- und Wissenschaftsbereichen.

Ein einführendes Kapitel behandelt Grundbegriffe des Messwesens, der Geodäsie und der Ausgleichungsrechnung. Messen ist heute weitgehend mit einer parallel laufenden funktechnischen Übertragung von Daten verbunden. Nahezu alle Bereiche des Spektrums der elektromagnetischen Wellen kommen heute bei geodätischen Messverfahren und dem Datenfunk zur Anwendung. Dieser Thematik widmet sich das 2. Kapitel. In Kapitel 3-5 werden die Instrumente und Messverfahren für die Richtungs- und Streckenmessung dargestellt. Neuere Instrumente arbeiten mit elektrischen Sensoren und lassen daher von der Aufnahme der Messwerte im Feld bis zur Übernahme in Informationssysteme oder bis zur Herstellung von Karten und 
Plänen weitgehend automatische Arbeitsprozesse zu. Höchste Entwicklungsstufen der Messsysteme haben Robotereigenschaften und werden daher Messroboter genannt. Die folgenden Kapitel 6-8 führen den Inhalt konsequent weiter, indem von den Messverfahren zu den Berechnungsmethoden übergegangen wird. Sie behandeln Koordinatensysteme und das Berechnen von Lagepunkten in Bezug auf diese, wobei Kapitel 6 als einfache Einführung dient. Kapitel 9 befasst sich mit der 3DTrägheitsnavigation. Die hier beschriebenen Strap-down-Systeme dienen einerseits der Navigation und kommen andererseits zunehmend zum Einsatz, wenn Messdaten in bewegten Fahrzeugen erfasst werden sollen. In Kapitel 10 wird gezeigt, dass insbesondere die Satellitenverfahren heute ein weites Spektrum neuer Aufgaben eröffnen, das von Arbeiten des amtlichen Vermessungswesens über die Ingenieurvermessung bis zur Navigation von Land- und Wasserfahrzeugen reicht. Auf diesem Gebiet vollzieht sich zur Zeit in der Geodäsie ein revolutionärer Schritt: der Übergang von den vermarkten zu den nicht vermarkten Festpunktfeldern. Letztere werden heute durch die permanent betriebenen Satelliten-Referenzstationen in Referenzstationsnetzen zur Verfügung gestellt. Die Kapitel 11-15 befassen sich mit dem weiten Spektrum der Höhenmessverfahren. Da heute der Vermessungsingenieur nicht nur nationale, sondern auch vielfach länderübergreifende Aufgaben lösen muss, behandelt das 16. Kapitel einführend den historischen Aufbau sowie die Weiterentwicklung nationaler, kontinentaler und globaler Festpunktfelder. Kapitel 17 beschreibt die Aufnahme großmaßstäbiger und topographischer Karten. Der Automatisierung sowie der Erfassung und Verarbeitung der Messwerte wird hier besondere Bedeutung gegeben. Neu wird in Kapitel 18 auf Grundbegriffe der Navigation und der Location Based Services eingegangen. Wie die Methoden der Geodäsie in anderen Ingenieurbereichen und wissenschaftlichen Disziplinen eingesetzt werden können, ist Gegenstand von Kapitel 19. Da es sich hier um ein sehr breites Anwendungsgebiet handelt, kann die Vorgehensweise nur exemplarisch gezeigt werden.

Kapitel 16 wurde weitgehend von Herrn Prof. Dr. Wolfgang Augath und Kapitel 18.2 von Herrn Ass. Prof. Dr. Günther Retscher formuliert.

Besonderer Dank gebührt meiner Frau Mechthild, die mit viel Mühe und Geduld die Texte mit dem Satzsystem $\mathrm{LT}_{\mathrm{E}} \mathrm{X}$ in die digitale Form übertragen hat. Dank gebührt außerdem Frau Dr. Irene Zimmermann; sie hat nicht nur die Texte durchgesehen, sondern auch Texte und Bilder in ein neues Format gebracht. Herrn Dr. Manfred Karbe, der bis Ende 2004 das Buch und auch diese Neuauflage für den Verlag betreute, möchte ich für die langjährige Zusammenarbeit und viele wertvolle Hinweise danken. 